\title{
Article \\ Classification of Prefrontal Cortex Activity Based on Functional Near-Infrared Spectroscopy Data upon Olfactory Stimulation
}

\author{
Cheng-Hsuan Chen ${ }^{1,2}{ }^{\oplus}$, Kuo-Kai Shyu ${ }^{1}$, Cheng-Kai Lu ${ }^{3}{ }^{\oplus}$, Chi-Wen Jao ${ }^{4}$ and Po-Lei Lee ${ }^{1, *}$ \\ 1 Department of Electrical Engineering, National Central University, No.300, Zhongda Rd., Zhongli District, \\ Taoyuan City 32001, Taiwan; $048853 @$ mail.fju.edu.tw (C.-H.C.); kkshyu@ee.ncu.edu.tw (K.-K.S.) \\ 2 Department of Electrical Engineering, Fu Jen Catholic University, No.510, Zhongzheng Rd., \\ Xinzhuang District, New Taipei City 242062, Taiwan \\ 3 Institute of Health \& Analytics for Personalised Care, Universiti Teknologi PETRONAS, \\ Seri Iskander 32610, Perak, Malaysia; chengkai.lu@utp.edu.my \\ 4 Institute of Biophotonics, National Yang-Ming University, No.155, Sec. 2, Linong Street, Taipei 11221, Taiwan; \\ c3665810@ms24.hinet.net \\ * Correspondence: pllee@ee.ncu.edu.tw; Tel.: +886-3-4227151 (ext. 35132)
}

Citation: Chen, C.-H.; Shyu, K.-K.; Lu, C.-K.; Jao, C.-W.; Lee, P.-L. Classification of Prefrontal Cortex Activity Based on Functional Near-Infrared Spectroscopy Data upon Olfactory Stimulation. Brain Sci. 2021, 11, 701. https://doi.org/ $10.3390 /$ brainsci11060701

Academic Editor: Dimiter Prodanov

Received: 11 March 2021

Accepted: 19 May 2021

Published: 26 May 2021

Publisher's Note: MDPI stays neutral with regard to jurisdictional claims in published maps and institutional affiliations.

Copyright: () 2021 by the authors. Licensee MDPI, Basel, Switzerland. This article is an open access article distributed under the terms and conditions of the Creative Commons Attribution (CC BY) license (https:// creativecommons.org/licenses/by/ $4.0 /)$.

\begin{abstract}
The sense of smell is one of the most important organs in humans, and olfactory imaging can detect signals in the anterior orbital frontal lobe. This study assessed olfactory stimuli using support vector machines (SVMs) with signals from functional near-infrared spectroscopy (fNIRS) data obtained from the prefrontal cortex. These data included odor stimuli and air state, which triggered the hemodynamic response function (HRF), determined from variations in oxyhemoglobin $(\mathrm{oxyHb})$ and deoxyhemoglobin (deoxyHb) levels; photoplethysmography (PPG) of two wavelengths (raw optical red and near-infrared data); and the ratios of data from two optical datasets. We adopted three SVM kernel functions (i.e., linear, quadratic, and cubic) to analyze signals and compare their performance with the HRF and PPG signals. The results revealed that oxyHb yielded the most efficient single-signal data with a quadratic kernel function, and a combination of HRF and PPG signals yielded the most efficient multi-signal data with the cubic function. Our results revealed superior SVM analysis of HRFs for classifying odor and air status using fNIRS data during olfaction in humans. Furthermore, the olfactory stimulation can be accurately classified by using quadratic and cubic kernel functions in SVM, even for an individual participant data set.
\end{abstract}

Keywords: functional near-infrared spectroscopy; olfaction; hemoglobin response function; support vector machine; classification; machine learning technique; prefrontal cortex

\section{Introduction}

Support vector machines (SVMs) help classify human data from functional nearinfrared spectroscopy (fNIRS) signals, and this method has novel applications [1,2], including a recently developed neuroimaging method for evaluating neural activity signals in brain cortical regions [3]. The classification accuracy of the eyes-open paradigm is $77.0 \%$; that of the eyes-closed paradigm is $75.6 \%$ [4]. The performances and outcomes of two linear classifiers reportedly benefit from shrinkage linear discriminant analysis. fNIRS can be performed with the eyes open or closed during the activation of the prefrontal cortex (PFC) during a mental arithmetic task [5]. A three-choice system-paced single trial with separate mental arithmetic and mental signing tasks in the no-control state has been developed, and a linear classifier achieved overall classification accuracy of $56.2 \%$ [6]. Numerous studies have reported that an SVM algorithm using fNIRS signals may have low accuracy. Furthermore, studies on olfaction have extensively used SVMs or other algorithms [7-10]. However, few studies have used fNIRS signals to measure olfactory stimuli or the air state with an SVM algorithm. 
fNIRS is used to investigate brain activity in healthy individuals, whose PFCs are stimulated through an olfactory stimulation task, and odorant intensity affects the hemodynamic response function (HRF) of oxyHb concentration [11,12]. Olfactory stimulation with an olfactometer using essential oils reportedly increases oxyHb levels and induces physiological and psychological relaxation [13]. Activation with strong fNIRS signals of the HRF in the PFC indicated olfactory stimulation when neonates were fed breast milk [14,15]. Using several odorants, Azuma et al. reported significant PFC activity during olfactory stimulation among patients with multiple chemical sensitivity (MCS) [16]. They reported olfactory stimulation by two odorants (sweet and fecal) at three concentrations (zero, odor recognition threshold, and normal perceived odor level) among MCS patients after fNIRS [17]. On identifying PFC activation due to olfactory stimulation, Azuma et al. reported that fNIRS is a valuable method for human testing [18].

Arterial blood oxygen saturation (SpO2) is a noninvasive dermal measurement, and photoplethysmography (PPG) signals of arterial pressure waves can be recorded at two wavelengths through fNIRS [19-21]. The pulse signal is measured using a light source with two wavelengths, red (PPG-R) and near-infrared (PPG-IR), and an optoelectronic detector detects the levels of light transmitted and reflected by human tissue under illumination [22].

The PPG waveform involves peak-to-peak amplitudes and has a pulsatile (AC) physiological waveform attributed to changes in the cardiac cycle from pumping a particular volume of blood with each baseline heartbeat and a gradually varying (DC) waveform along with various lower frequency components in venous blood, skin, and tissue [23,24]. The pulse signal ratio comprises two wavelengths (PPG-R and PPG-IR) of AC/DC and constitutes dbRatio data.

We used three SVM kernel functions (i.e., linear, quadratic, and cubic) to analyze and classify fNIRS signal data for olfactory activity. Signals were acquired from the PFC, where olfactory activity occurs. We acquired and transformed optical raw data to obtain a hemodynamic response (oxyHb and deoxyHb), PPG signal (PPG-R and PPG-IR), and $\mathrm{dbRatio}$ data, and then we used them to extract data features. The results of the preliminary trials using three kernel functions for SVM training are presented as performance accuracy and receiver operating characteristic (ROC) curves. The present SVM analysis of the HRF could serve as a novel method to classify odor and air status in human olfaction by using fNIRS.

\section{Materials and Methods}

\subsection{Participants}

Nine healthy individuals (seven male and two female; average age, 28 years) volunteered to participate in this study. The study design was explained to all participants. They had normal olfactory abilities and could smell lavender. Each was seated in a comfortable armchair. They heard white noise over earphones to reduce the effects of sound from the olfactometer; then, a bottle containing an odorant was opened.

\subsection{System Architecture}

The discussion of the system architecture (Figure 1a) is divided into three sections. The first section involves the acquisition and storage of data with an embedded NIRS device and a laptop computer, which monitor signals over the PFC in real time over two channels. The second section describes an experiment performed using lavender as an olfactory stimulus presented via an oxygen mask with an attached olfactometer and with an informative message relayed to the participants using Presentation software (Neurobehavioral Systems Inc., Berkeley, CA, USA). The third section describes the data analysis. Application of the raw data is discussed in two sections: One describes the incorporation of the raw data into MATLAB software to determine values estimating the percentage of oxygen bound to $\mathrm{Hb}$; the other describes the analysis of the fNIRS signals in Homer2 to obtain estimates of brain activity. 


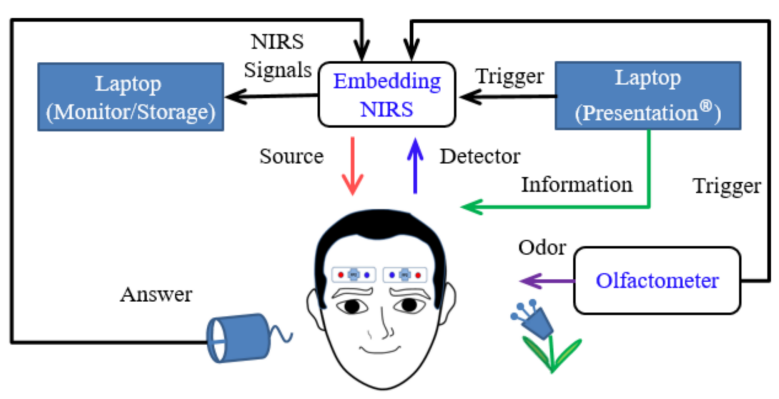

(a)

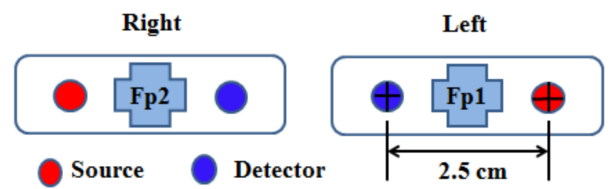

(b)

Figure 1. (a) Schematic of the study's fNIRS system design. (b) Optode placement and channel location on the prefrontal cortex at reference points Fp1 and Fp2, in accordance with the International 10-20 System of placement. This system comprises two sources and two detectors, and each source-detector pair is separated by $2.5 \mathrm{~cm}$.

\section{3. fNIRS Analysis}

We developed a flexible and simple NIRS protocol for monitoring brain activity. Our mainboard microcontroller, the STMicroelectronics Cortex-M4 Core Chip STM32F429, and daughter boards, AFE4490 models, were used for fNIRS data acquisition. The microcontroller functions at a $180 \mathrm{MHz}$ processor clock frequency. The system had 21 communication interfaces including six serial peripheral interfaces (SPIs) and four universal asynchronous receiver/transmitters (UARTs), which could be configured through software through general-purpose input/output (GPIO). We used SPIs to read the analog-to-digital converter values and raw data transmitted by the microcontroller UART for storage on the laptop computer. The GPIO received triggers from the olfactometer during the experiment.

To minimize development cost and time, we used shelf components including customized printed circuit boards and two D-sub miniature connectors compatible with the Nellcor MAXN sensor. The light sensor has two sources, NIR and red light-emitting diodes (LEDs), that transmit light through the PFC. Unabsorbed light is received by a photodetector; the sample rate was $500 \mathrm{~Hz}$ in the channel. We used a Texas Instruments Low-Cost Pulse Oximeter with an AFE 4900 analog chip to detect brain activity. The AFE4490's internal timer initiates the LED; the transceiver causes the light source to flash and detects the brain signal fed back.

\subsection{Optode Settings}

The LED emitter-detector pairs of two channels were positioned on the PFC to detect olfactory activity and rest signals, with two channels separated at the left and right side; the emitter-to-detector distance was $2.5 \mathrm{~cm}$. The emitter had two wavelengths ( $\lambda \mathrm{R}$ and $\lambda \mathrm{NIR}$ ), 660 and $960 \mathrm{~nm}$. The optode placement and channel location at Fp1 and Fp2 represent the reference points of the International 10-20 System (Figure 1b).

\subsection{Stimulus Paradigm}

The odor stimulus baseline was $35 \mathrm{~s}$, olfactory stimulant time was $12 \mathrm{~s}$, and rest time was $30 \mathrm{~s}$; each trial comprised 16 blocks with a $20 \mathrm{~s}$ interval between blocks. Participants received a $17 \mathrm{~s}$ informative message initiated $5 \mathrm{~s}$ before the stimulus and terminated $12 \mathrm{~s}$ after, and they were given $25 \mathrm{~s}$ to answer the questions (Figure 2). These trials employed the oddball paradigm design, with a $12 \mathrm{~s}$ stimulus (opening bottles), which was a lavender odorant or distilled water; the stimuli were assigned at a 50:50 ratio. The stimulus block assignment followed the pattern 10101001, where 1 was lavender odor and 0 was water. The first prompt on the laptop computer screen read "Prepare to smell" for $17 \mathrm{~s}$, and the second read "Did you smell?" after the stimulus. The participants in all blocks had $25 \mathrm{~s}$ to answer the question using a mouse. The participants were informed beforehand of the experimental paradigm about olfactory stimulant with air and odor and texts on computer screen but not about the regularity whole blocks. 


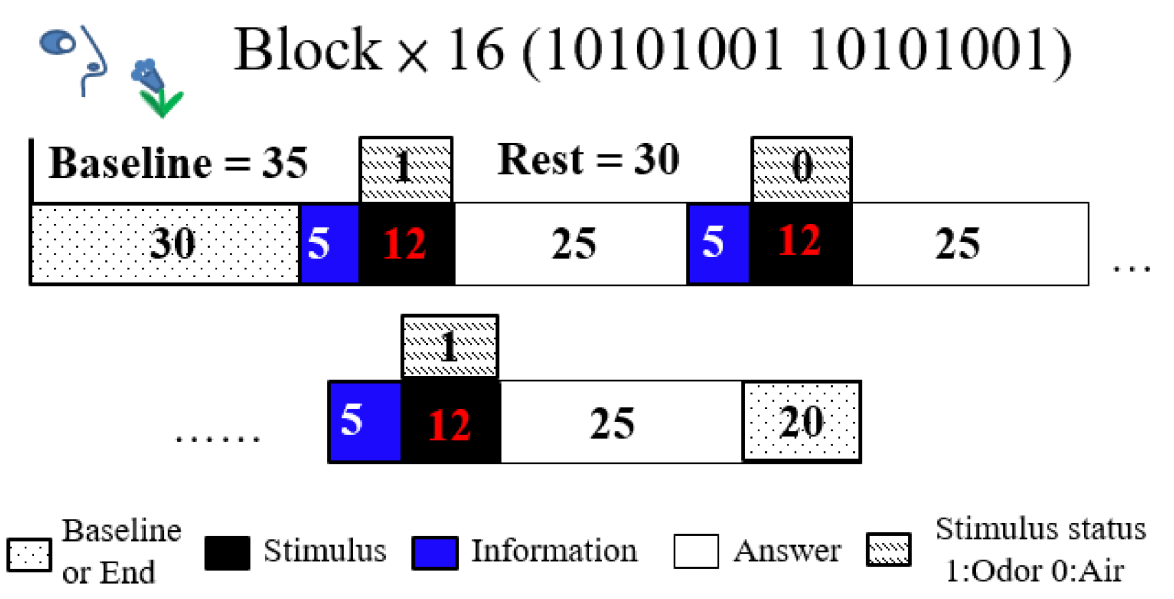

Figure 2. Schematic representation of the experimental paradigm: A block comprising a $12 \mathrm{~s}$ stimulus and a $30 \mathrm{~s}$ rest period, along with a $35 \mathrm{~s}$ baseline and $20 \mathrm{~s}$ termination period.

\subsection{Olfactory Delivery System}

The olfactometer (ETT, Hershey, PA, USA) used for the lavender odorant was delivered as a fully automated system consisting of a set of Teflon tubing that transported odor and air, using an air pump, through an array of odorant chambers with solenoid valves to both nostrils of the participants. The timing and termination of odorant delivery were automated in accordance with the stimulus paradigm. During data acquisition, clean, moisturized compressed room air with or without an odorant was delivered through the tubing into an oxygen mask approximately $3 \mathrm{~cm}$ from the participant's nose at a constant air flow rate of $8 \mathrm{~L} / \mathrm{min}$. The lavender intensity was set by diluting lavender oil to $2.0 \%$ intensity.

\subsection{Signal Processing and Data Acquisition}

Signal processing was performed in four stages (Figure 3): first, raw data were acquired; second, raw data were filtered, transforming the data into a hemodynamic response and PPG signals, and classifying the data into blocks; third, features were extracted from all data and the training data were labeled; fourth, classification and data statistics were performed using the SVM.

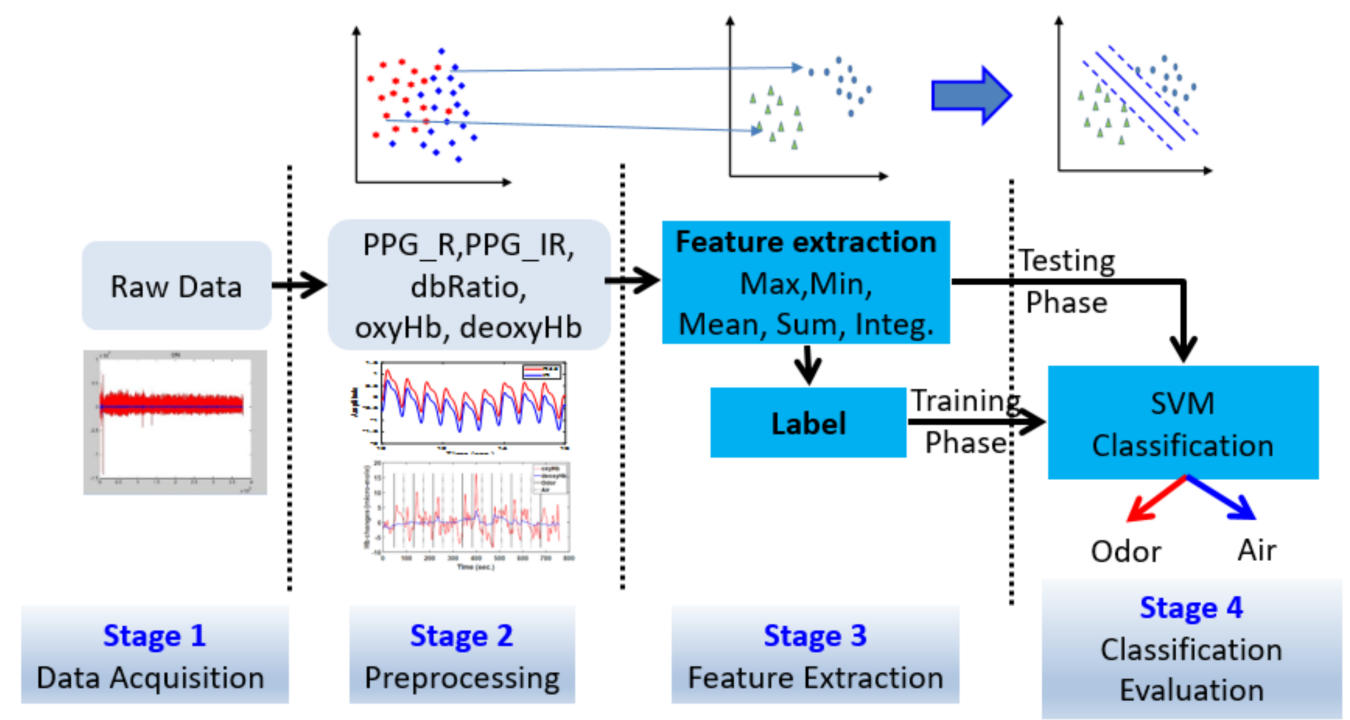

Figure 3. Flow chart of signal processing using the present machine learning approach to separate odor and air classes through photoplethysmography (PPG-R, PPG-IR, and dbRatio) and hemoglobin response function (including $0 x y H b$ and deoxyHb) signal data. 
To determine the hemodynamic response from raw data, specified red and infrared wavelengths of light were transmitted to the scalp and then scattered through the cortex, where chromophores of oxyHb and deoxyHb, which absorb some NIR light, are present. Brain activity was detected by the detectors, raw intensity was recorded from the data transferred from the detectors, and the voltage was measured.

Signal processing filters were applied to the raw data. Thereafter, the data were transformed into hemodynamic responses; PPG signals and blocks of time were added to create block data in the second stage. The intensity data were then used to determine changes in the optical density (delta-optical density). The Homer2 software tool was used to analyze the hemodynamic responses of data associated with oxy $\mathrm{Hb}$ and deoxyHb concentrations. The SpO2 codes could be used to determine PPG curves in two wavelengths, red and infrared (PPG-R, PPG-IR), and are derived from the dbRatio, as follows [25-27]:

$$
\mathrm{dbRatio}=\frac{\operatorname{Red}(\mathrm{AC} / \mathrm{DC})}{\operatorname{IR}(\mathrm{AC} / \mathrm{DC})}
$$

where AC and DC are the peak-to-peak amplitudes and baseline of the PPG pulse, respectively.

Data were recorded in $33 \mathrm{~s}$ blocks at a sample rate of $500 \mathrm{~Hz}$; those data were the same as those recorded with a stimulus time of $3 \mathrm{~s}$ before and $30 \mathrm{~s}$ after. The locations of changes in the PPG-R, PPG-IR, dbRatio, oxyHb, and deoxyHb signals in each channel were observed in nine participants. The dbRatio signals were recorded for $33 \mathrm{~s}$, as shown in Figure $4 \mathrm{~b}$.

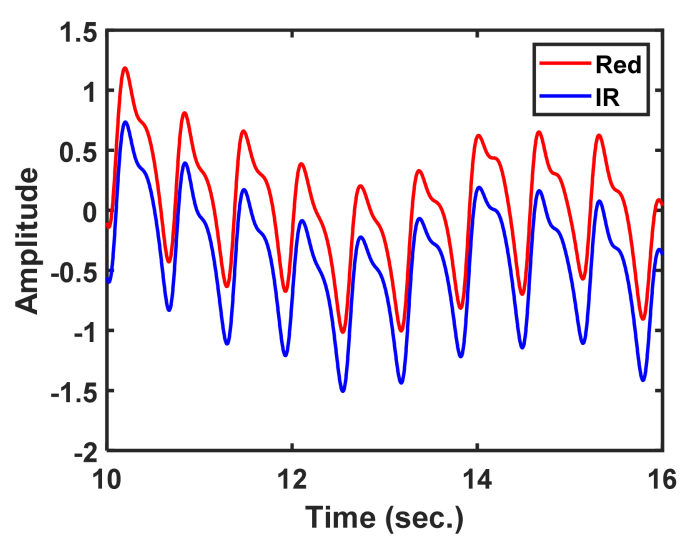

(a)

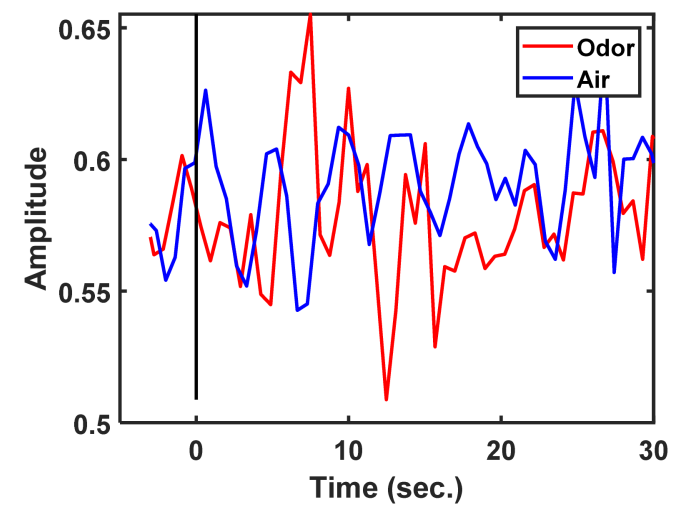

(b)

Figure 4. Functional near-infrared spectroscopy measurement of the prefrontal cortex: (a) raw photoplethysmography (PPG) PPG-R and PPG-IR signals, (b) 33 s block data in which the dbRatio waveforms have an odor and air status.

\subsection{Hemodynamic Response Function}

Neuronal activation can be convolved with an HRF in the brain obtained through vascular imaging modalities based on stimuli and assessed through functional magnetic resonance imaging and fNIRS [28-30]. Numerous studies have attempted to use this hemodynamic response to better understand the mechanisms underlying neurovascular and neurometabolic coupling. The fNIRS signal intensity is altered during brain stimulation. $\mathrm{OxyHb}$ is detected through an increase in its concentration. The opposite is true of deoxyHb, which can be detected through a reduction in its concentration during brain activation. Increases in neural activity beyond the baseline level increase oxy $\mathrm{Hb}$ and decrease deoxyHb levels [31,32], as show Figures 5 and 6 . In the present study, fNIRS was performed to assess the changes in the HRF of oxyHb and deoxyHb concentrations in accordance with the modified Beet-Lamberts law (BLL). The BLL algorithm in Homer2 software was used such that optical data represented the $\mathrm{Hb}$ concentration $[33,34]$. 


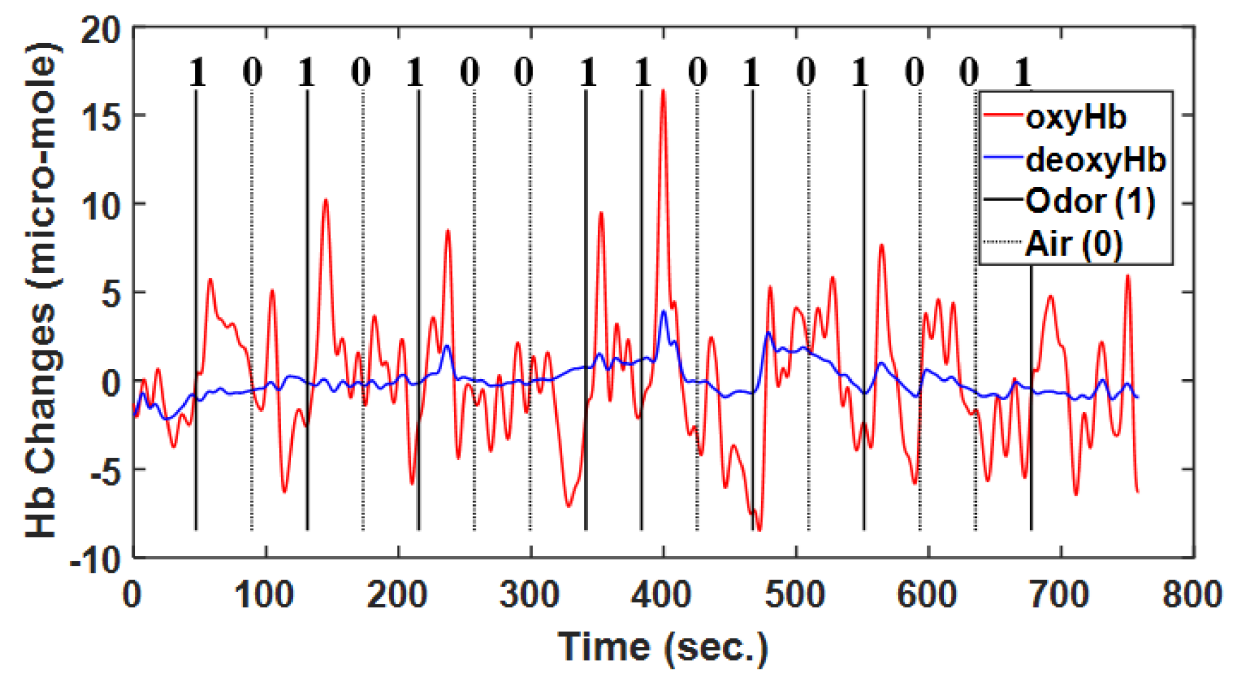

Figure 5. Typical example of raw hemodynamic response function including oxyHb and deoxyHb data, with air and odor as the stimuli, in accordance with the experimental paradigm.

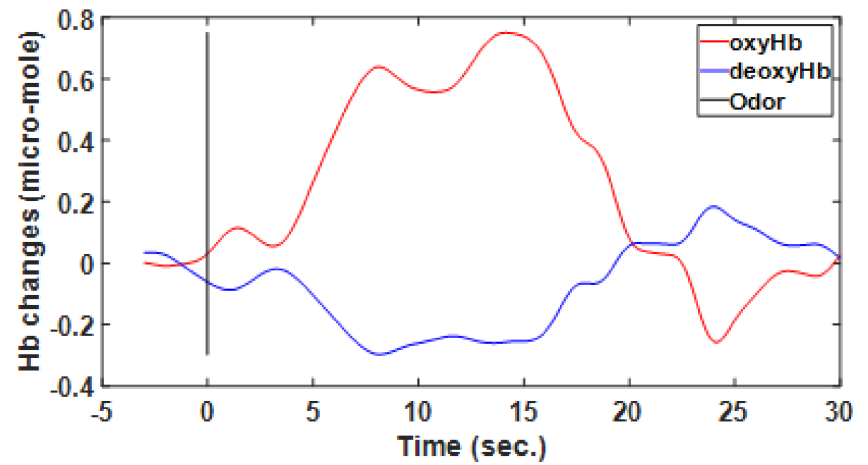

(a)

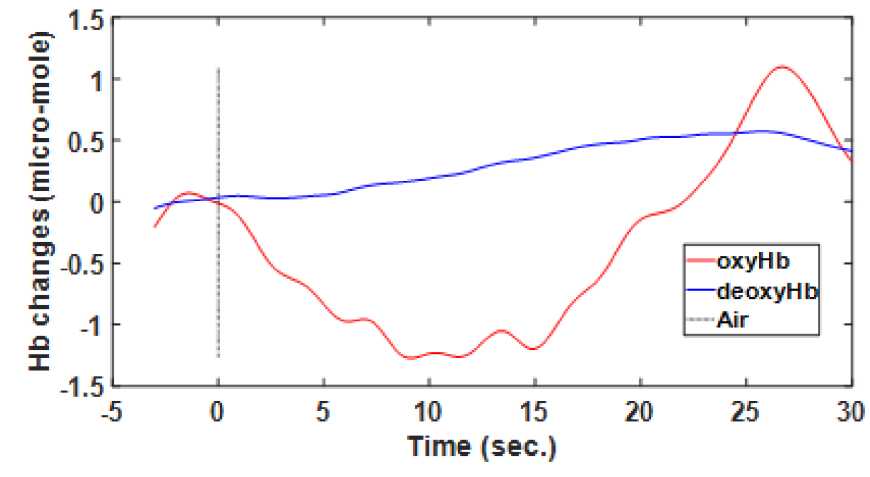

(b)

Figure 6. Hemodynamic response of one participant determined through functional near-infrared spectrometry. Subplots show the averaged results for the region of interest from among eight blocks wherein odor (a) and air (b) were used as individual stimuli.

\subsection{Feature Extraction}

Feature data during signal processing were extracted during the third stage. We harnessed the following statistical properties of the five time-domain signals,

PPG-R, PPG-IR, dbRatio, oxyHb, and deoxyHb, as follows:

- Maximum (Max.);

- Minimum (Min.);

- Average (Mean.);

- Amount (Sum.);

- Trapezoidal numerical integration (Integ.).

These feature data have five signals and five features, separated by the two stimulus states of odor and air data, and status labels were assigned in a table for SVM training. Two types of data were used for training: a five-type signal yielding a single data point and a nine-type mixed signal yielding multiple data from a combination of two or three signals. The training results are listed as 14 types of data in Tables 1-3.

All the signal data were normalized before feature extraction using the following Z-Score formula [35]. 
Table 1. Support vector machines with linear kernel function; all signals were photoplethysmography signals (PPG-R, PPG-IR, and PPG-dbRatio) and hemodynamic response function (oxyHb and deoxyHb) signals. The superscript asterisk is the best in table of accuracy.

\begin{tabular}{ccccc}
\hline Type of Data & Accuracy (\%) & Sensitivity (\%) & Specificity (\%) & AUC \\
\hline PPG-R & 56.25 & 70.83 & 58.3 & 0.59 \\
PPG-IR & 47.92 & 72.92 & 33.33 & 0.47 \\
PPG-dbRatio & 43.75 & 4.16 & 100 & 0.42 \\
oxyHb & 44.79 & 0 & 39.58 & 0.37 \\
deoxyHb & $58.33 *$ & 91.67 & 47.92 & 0.64 \\
PPG-R and PPG-IR & 54.17 & 77.08 & 4.17 & 0.59 \\
HRF (oxyHb and deoxyHb) & 40.63 & 97.92 & 12.5 & 0.32 \\
PPG signals & 43.75 & 91.67 & 10.42 & 0.4 \\
deoxyHb and PPG signals & 39.58 & 91.67 & 14.58 & 0.39 \\
oxyHb and PPG signals & 40.63 & 91.67 & 2.08 & 0.34 \\
HRF and PPG (-R-dbRatio) & 34.38 & 100 & 10.42 & 0.32 \\
HRF and PPG (-IR-dbRatio) & 37.5 & 97.92 & 4.17 & 0.32 \\
HRF and PPG (-R-IR) & 38.54 & 97.92 & 0.33 \\
\hline
\end{tabular}

Table 2. Support vector machines with quadratic kernel function; all signals were photoplethysmography signals (PPG-R, PPG-IR, and PPG-dbRatio) and hemodynamic response function signals (oxyHb and deoxyHb). The superscript asterisk is the best accuracy in table.

\begin{tabular}{ccccc}
\hline Type of Data & Accuracy (\%) & Sensitivity (\%) & Specificity (\%) & AUC \\
\hline PPG-R & 47.92 & 66.67 & 52.08 & 0.56 \\
PPG-IR & 52.08 & 22.92 & 85.42 & 0.5 \\
PPG-dbRatio & 56.25 & 29.17 & 89.58 & 0.59 \\
oxyHb & $87.5 *$ & 100 & 97.92 & 0.98 \\
deoxyHb & 48.96 & 60.42 & 54.17 & 0.53 \\
PPG-R and PPG-IR & 55.21 & 52.08 & 62.5 & 0.54 \\
HRF (oxyHb and deoxyHb) & 83.33 & 95.83 & 79.17 & 0.92 \\
PPG signals & 57.29 & 54.17 & 64.58 & 0.57 \\
deoxyHb and PPG signals & 52.08 & 72.92 & 50 & 0.55 \\
oxyHb and PPG signals & 70.83 & 75.58 & 0.77 \\
HRF and PPG (-R-dbRatio) & 67.71 & 91.67 & 66.67 & 0.69 \\
HRF and PPG (-IR-dbRatio) & 69.79 & 77.08 & 56.25 & 0.74 \\
HRF and PPG (-R-IR) & 69.79 & 75 & 68.75 & 0.75 \\
HRF and PPG signals & 71.88 & 68.75 & 0.72 \\
\hline
\end{tabular}

Table 3. Support vector machines with cubic kernel function; all signals were photoplethysmography signals (PPG-R, PPG-IR, and PPG-dbRatio) and hemodynamic response function signals (oxyHb and deoxyHb). The superscript asterisk is the best accuracy in table.

\begin{tabular}{ccccc}
\hline Type of Data & Accuracy (\%) & Sensitivity (\%) & Specificity (\%) & AUC \\
\hline PPG-R & 52.08 & 20.83 & 85.42 & 0.5 \\
PPG-IR & 51.04 & 62.5 & 52.08 & 0.54 \\
PPG-dbRatio & 55.21 & 83.33 & 45.83 & 0.62 \\
oxyHb & $84.38 *$ & 89.58 & 83.33 & 0.92 \\
deoxyHb & 40.63 & 0 & 100 & 0.38 \\
PPG-R and PPG-IR & 46.88 & 93.75 & 12.5 & 0.48 \\
HRF (oxyHb and deoxyHb) & 79.17 & 91.67 & 66.67 & 0.76 \\
PPG signals & 62.5 & 58.33 & 66.67 & 0.59 \\
deoxyHb and PPG signals & 52.08 & 72.92 & 47.92 & 0.57 \\
OxyHb and PPG signals & 69.79 & 75 & 52.92 & 0.73 \\
HRF and PPG (-R-dbRatio) & 58.33 & 68.75 & 52.08 & 0.65 \\
HRF and PPG (-IR-dbRatio) & 65.63 & 89.58 & 64.58 & 0.65 \\
HRF and PPG (-R-IR) & 62.5 & 66.67 & 43.75 & 0.67 \\
HRF and PPG signals & 64.58 & 66.67 & 0.65 \\
\hline
\end{tabular}




\subsection{Support Vector Machine}

In the fourth stage of signal processing, the SVM was trained to classify data points. In particular, the SVM helped to identify the hyperplane, which provides a maximum margin between the plane surface and positive and negative points. This separating hyperplane was used as a frontier for classification, which becomes optimal at closed points, and is referred to as an SVM. The SVM was constructed by solving a dual optimization problem as follows:

$$
\begin{array}{ll}
\text { maximize } & \sum_{i=1}^{N} a_{i}-\frac{1}{2} \sum_{i, j}^{N} a_{i} a_{j} y_{i} y_{j} K\left(x_{i} x_{j}\right) \\
\text { subject to } & \sum_{i=1}^{N} a_{i} y_{i}=0,0 \leq a_{i} \leq C \forall i=1, \ldots N .
\end{array}
$$

The coordinates $\left(x \_i, y \_i\right)$ are training samples, and $C$ is the penalty parameter for the slack variable that should be minimized [36]. The maximized equation of $K\left(x_{-} i, x_{-}\right)$is the kernel function used to embed the training samples in an n-dimensional space. The kernel function affects the accuracy of the SVM classifier, which largely depends on the type used; several kernel functions are available for nonlinear mapping of input patterns. We used the polynomial kernel function to train and test our SVM classifier. Nonlinear transformation with the kernel function was performed as follows:

$$
\text { Polynomial } K\left(x_{i}, x_{j}\right)=\left(1+x_{i} x_{j}\right)^{\rho}
$$

where $\rho$ is the order of the polynomial.

The kernel function parameter $\rho$ in Equation (4) and the margin-loss trade-off $C$ in Equation (3) were considered to determine the optimal SVM performance and obtain an optimal $C$ that the classifier can predict from unknown data or testing data. Optimal values of parameters were obtained through cross-validation using a grid search algorithm. The SVM was originally a binary classifier; therefore, we intended to separate the status of the two stimuli, odor and air [37,38].

\subsection{Classification Evaluation}

The accuracy, specificity, and sensitivity of classification performance were assessed using the true and false positive (TP, FP) rates and true and false negative (TN, FN) rates. Accordingly, the accuracy, specificity, and sensitivity were determined as follows [39]:

$$
\begin{gathered}
\text { Accuracy }=\frac{\mathrm{TP}+\mathrm{TN}}{\mathrm{TP}+\mathrm{TN}+\mathrm{FP}+\mathrm{FN}} \\
\text { Sensitivity }=\frac{\mathrm{TP}}{\mathrm{TP}+\mathrm{FN}} \\
\text { Specificity }=\frac{\mathrm{TN}}{\mathrm{TN}+\mathrm{FP}}
\end{gathered}
$$

Classification performance was analyzed on the basis of the hemodynamic response curve data, include PPG signals (PPG-R, PPG-IR, and dbRatio) and hemodynamic response signals (oxyHb and deoxyHb; Tables 1-3).

\subsection{ROC Curve Analysis}

ROC curves help determine the potential of various statistical methods. An ROC curve is a plot of a test's TP rate (sensitivity) versus its FP (specificity). Thus, an ROC curve describes the trade-off between the sensitivity and specificity of the test, based on changes in the "negative" and "positive" results. The area under the ROC curve (AUC) is a quantitative measure of selectivity (e.g., 1.0 for the best selectivity; 0.5 for the worst selectivity) and relative accuracy values (ratio of TP and TN to all test samples). It is a function of both the sensitivity and specificity of a test, considering the entire range of errors. We performed ROC analysis of our SVM-based classification [40-42]. 


\subsection{Data Analysis}

Data were analyzed using the SVM algorithm and MATLAB 9.5 (The MathWorks Inc., Natick, MA, USA).

\section{Results}

The PFC hemodynamic responses of nine participants were analyzed. There were 9 subjects with a total of 25 experiments, and data were available from each of the 23 experiments, one from each experiment. Two of the subjects had two experiments each for a total of 4 times, and the remaining seven had three experiments each for a total of 21 times. We excluded two experiments from the seventh because the smell of lavender made the subjects doze off easily and caused no signal. The remaining 23 trials were included in the training dataset, and 16 blocks (eight odor and eight air blocks) were included at random in the trial. The training data included 368 block datasets, with 184 data for odor or air status. On analyzing the overall datasets, 16,500 data points were in each $33 \mathrm{~s}$ block $(500 \mathrm{~Hz})$.

Figure 7a displays the performance of a single signal of oxyHb on the ROC curve, for which the AUC of the quadratic and cubic kernel functions was 0.98 and 0.92 , respectively. Figure $7 \mathrm{~b}$ displays five signals of features in the training set for three kernel functions, where the performance of the ROC curve and SVM kernel function (quadratic and cubic) of the AUC were 0.72 and 0.65 , respectively.

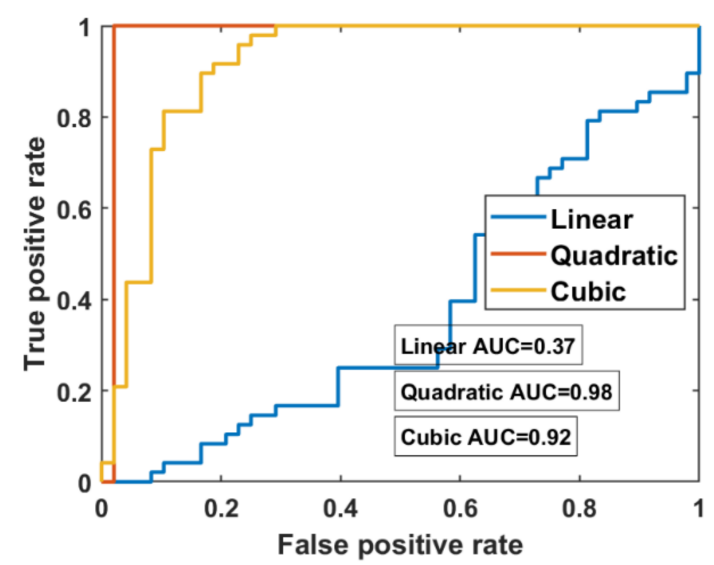

(a)

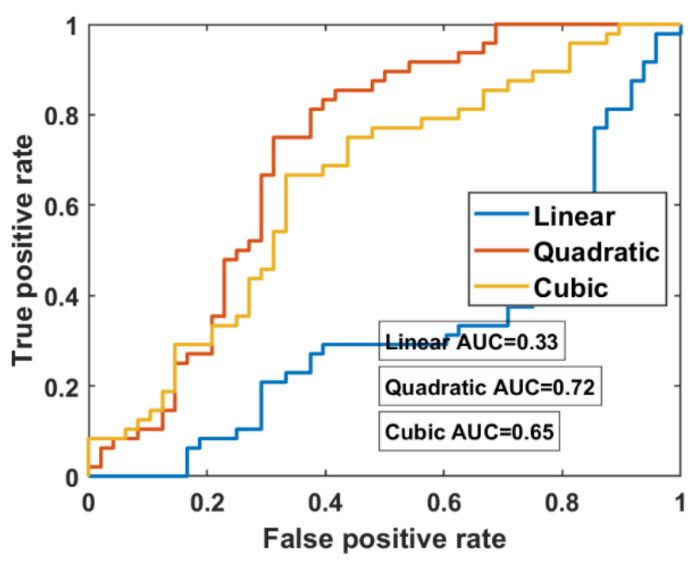

(b)

Figure 7. Receiver operating characteristic curves of signals of a dataset with performance of three kernels for an SVM classifier: (a) single signal of oxyHb using the quadratic kernel with an area under the curve of 0.998 , and (b) combination of five signals with the optimal area under the curve of 0.72 with the cubic function.

The ROC curve illustrates the performance of the classification model for optimal features, determined from the trade-off between sensitivity and specificity. The AUC quantifies the overall potential of the classifier to distinguish between stimuli. An ideal classifier has an AUC of 1, and a random classifier has an AUC of 0.5. Therefore, the larger the AUC, the better the performance of the classifier.

The specificity and sensitivity values were obtained by averaging the correspondence across one or more data sets. A comparison of results for single signals and multiple signals for three SVM kernel functions is depicted in Tables 1-3.

Figure 8 illustrates the single- and multi-signal accuracy of three kernel functions. Types 1 to 5 are single-signal data; Types 6 to 8 are two or three signals integrated into a table for the SVM training phase; Types 9 to 13 are multiple signals with four pieces of fNIRS data; Type 14 is integrated data from five signals. In detail, the types of signal data were as follows: (1) PPG-R; (2) PPG-IR; (3) dbRatio; (4) OxyHb; (5) deoxyHb; (6) PPG-R and PPG-IR; (7) HRF (oxyHb and deoxyHb); (8) PPG-R, PPG-IR, and dbRatio; (9) PPG signals and deoxyHb; (10) PPG signals and oxyHb; (11) HRF, PPG-R, and dbRatio (excluding IR); 
(12) HRF, PPG-IR, and dbRatio (excluding PPG-R); (13) HFR, PPG-R, and PPG-IR signals (without dbRatio); and (14) multiple signals with five fNIRS data and HRF.

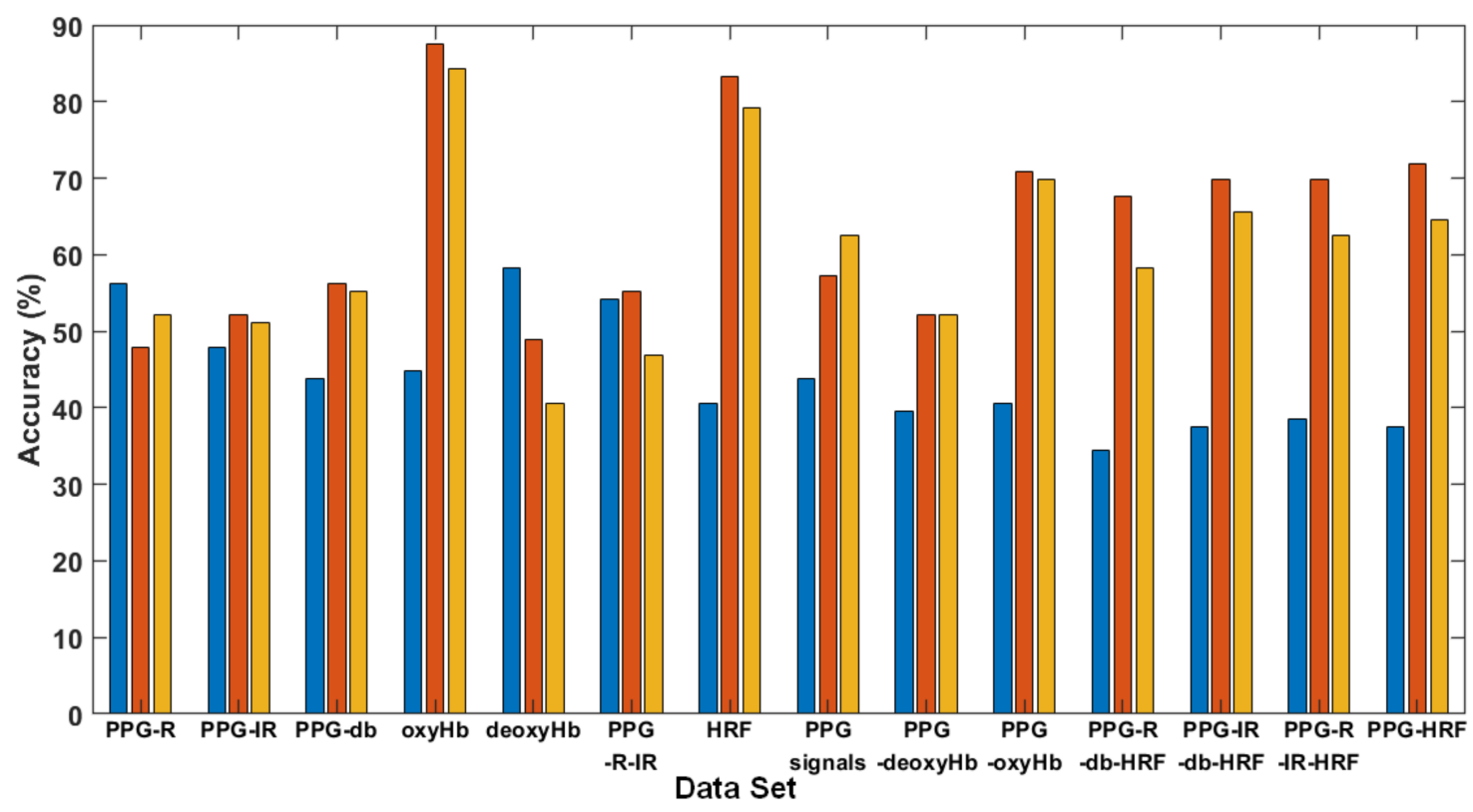

Figure 8. Accuracy comparison for 14 types of data and three kernel functions: linear, quadratic, and cubic.

As mentioned in the above tables, even in a smaller data set, the Quadratic or Cubic method revealed a superior performance in clustering than the linear method did (please refer to S7 in above table). By used linear method, the S7 of accuracy was only $50 \%$, and the accuracy of clustering was prompt raised to $100 \%$ or $68.75 \%$ by using Quadratic or Cubic methods.

The best performance of clustering is $\mathrm{S} 6$ and the accuracy was $56.25 \%, 97.92 \%$ and 95.83 by using Linear Quadratic and Cubic methods, respectively in the Table 4. Furthermore, our results evidenced that the olfactory stimulation in prefrontal cortex activity can be accurately classified by quadratic and cubic kernel functions in SVM, even for an individual participant data set.

Table 4. Accuracies for individual participants with three kernel functions.

\begin{tabular}{|c|c|c|c|c|c|}
\hline Participants & Linear Accuracy (\%) & Quadratic Accuracy (\%) & Cubic Accuracy (\%) & Block Number & Train-Test Ratio \\
\hline S1 & 65.63 & 90.63 & 84.38 & 32 & 91.3:8.7 \\
\hline S2 & 53.13 & 96.88 & 93.75 & 48 & $87: 13$ \\
\hline S3 & 56.25 & 90.63 & 90.63 & 32 & 91.3:8.7 \\
\hline $\mathrm{S} 4$ & 52.08 & 83.33 & 87.5 & 48 & $87: 13$ \\
\hline S5 & 48.44 & 90.63 & 76.56 & 48 & $87: 13$ \\
\hline S6 & 56.25 & 97.92 & 95.83 & 48 & $87: 13$ \\
\hline S7 & 50 & 100 & 68.75 & 16 & $95.7: 4.3$ \\
\hline S8 & 50 & 75 & 77.08 & 48 & $87: 13$ \\
\hline S9 & 54.17 & 89.58 & 81.25 & 48 & $87: 13$ \\
\hline Average & 53.99 & 90.51 & 83.97 & 40.9 & $89: 11$ \\
\hline
\end{tabular}

\section{Discussion}

We evaluated an innovative approach based on fNIRS signals for assessing olfactory signals in the PFC through SVM-based classification. Two HRF and PPG signals were evaluated using single or multiple signals from three SVM training kernel functions. The oxyHb of HRF displayed enhanced performance and accuracy. The fNIRS signal features were extracted for training, and these were primarily normal arithmetic features. 
We used the simple Max., Min., Mean., Sum., and Integ. Feature extraction methods to accelerate the operation for embedded systems. We attempted to identify the most efficient set of features from participants' brain activity $[43,44]$. Therefore, feature extraction from PPG and HRF signals was simplified through machine learning.

We used the Z-Score to normalize signals but disregarded the data processes that might influence accuracy. When the Z-Score was neglected (see Figure 9a), the oxyHb signals of accuracy with the cubic function dropped $84.38 \%$ to $53.13 \%$, but the quadratic is not effected instead $87.5 \%$ up to $94.79 \%$. The multi-signal (PPG and oxyHb) data resulted in accuracy of $70.83 \%$ which decreased to $59.38 \%$ with a quadratic function (Figure $9 b$ ). However, the Z-Score improved the accuracy results for two-signal data for the three kernel functions, as shown in Figure 9.

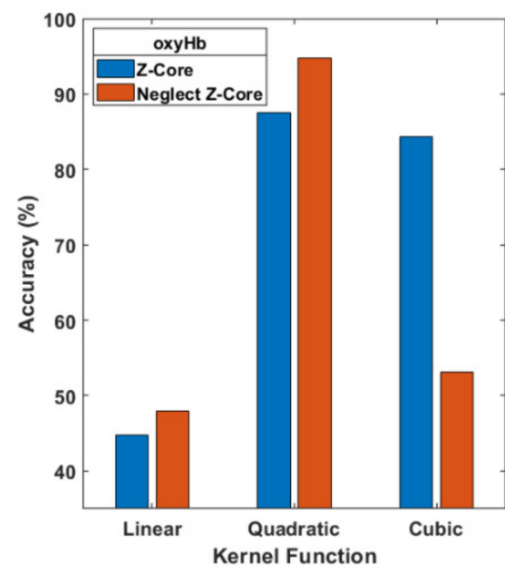

(a)

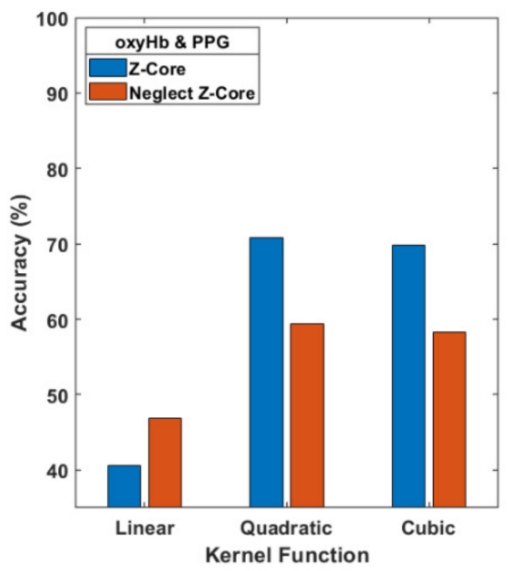

(b)

Figure 9. Comparison of the Z-Score and neglect Z-Score for single (a) and multiple signals (b) and three kernel functions.

We have separated two kind of data for train set and test set. The total data was 368 available that 23 trials for each was 16 blocks. The training data were comprised of 272 blocks and the test data were comprised of 96 blocks; the cross-validation data which uses the "fitcsvm" function in MATLAB software by default used 10-fold the amount of training data. The test data set was taken from subject 1 to 6 , each taking the second trial that the odor and air was eight blocks for every trial; the total was 96 blocks.

During the preparation of the training data, we used MATLAB software. Matrix transposition is an important step before feature extraction. Therefore, input data were arranged in a matrix of data acquired over time and recorded continuously in a column, and signal processing of the data in the column served as a stimulus block event. For example, the input data matrix dimensions were $16,500 \times 8$ (time points $\times$ blocks), which must be transposed to $8 \times 16,500$ (blocks $\times$ time points); otherwise, errors might be incurred during execution in MATLAB.

For sufficient training data, trial numbers from two to three trials were included for each participant; data from two participants who fell asleep during the experiment were excluded. In total, $727 \mathrm{~s}$ of trial data were acquired: $672 \mathrm{~s}$ constituted the toal time for 16 blocks (rest of $30 \mathrm{~s}$ and stimulus of $12 \mathrm{~s}$ ), and $35 \mathrm{~s}$ of baseline and $20 \mathrm{~s}$ at the end were added. Keep awake, those participants to small, a question showed on laptop $\left(\right.$ Presentation ${ }^{\circledR}$ ) and answered by mouse click. For related olfactory experiments, a shorter time may be preferable.

The fNIRS set of the optical (source-detector) pair at Fp1 and Fp2 (International 10-20 System) at the PFC is reasonable and facilitated the assessment of brain activity after the odor stimulus [11-18]. The olfactory complex comprises several areas, including the anterior olfactory nucleus, piriform cortex, olfactory tubercle, anterior cortical amygdaloid nucleus, periamygdaloid cortex, and entorhinal cortex, which receive inputs from the 
olfactory bulb and are present in a structurally distinct cortical region. The accessory olfactory bulb has medial and posterior cortical amygdaloid nuclei. Extrinsic axonal projections from the olfactory cortex to the orbital cortex, mediodorsal thalamic nucleus, hypothalamus, amygdala, and hippocampus are present [45]. The orbital cortex is a part of the PFC, brain area 13a of the posterior orbitofrontal cortex, and contains direct connections to the primary olfactory cortex unit [46].

Decreased olfactory function is common among patients with idiopathic Parkinson's disease [47] or Alzheimer's disease [48] and older individuals [49]. Olfactory function in humans plays important protective roles and contributes to interpersonal interactions, eating habits, and nutrient intake, which are severely altered in numerous patients with olfactory disorders [50]. Loss of olfactory function is present in approximately $50 \%$ of older individuals (65-80 years) and approximately $75 \%$ of those aged $\geq 80$ years [51]. An early diagnosis of olfactory dysfunction through fNIRS is thus recommended.

In future studies, we intend to investigate other features extracted from HRF and PPG signals. Furthermore, fNIRS may be using embedded in a mobile device with a data center which big data processing online. Further studies are required that predict olfactory dysfunction among participants with SVMs or other machine learning techniques with large clinical datasets.

\section{Conclusions}

This study reports the potential of using an SVM for training five types of single fNIRS signal data and nine types of multiple fNIRS signal data, extracting features regarding olfactory stimuli, yielding the original HRF and PPG signals from fNIRS raw data. We developed an fNIRS system that measures raw data at the PFC through olfactory stimulation.

Among three SVM kernels, the quadratic function was optimal for fNIRS signals, with average accuracy of $63.61 \%$. The cubicand linear kernel functions had average accuracy of $60.34 \%$ and $44.12 \%$, respectively. Therefore, we used the quadratic kernel function.

For 14 types of data, including five pieces of single-signal data using the quadratic kernel function, the accuracy of oxyHb was the highest at $87.5 \%$, whereas the accuracy for PPG-R was $47.92 \%$, lower than that of the others. Multiple oxyHb and PPG signals (PPG-R, PPG-IR, and dbRatio) with the quadratic kernel function resulted in an adequate accuracy of $>70.83 \%$. Type 11-14 resulted in an accuracy of $>67 \%$; however, Types 6 and 8 included PPG but excluded oxyHb, and the accuracy decreased to $55.21 \%$ and $57.29 \%$. Type 5, deoxyHb, resulted in the lowest accuracy, at $48.96 \%$. The oxyHb signal affected the performance accuracy of both the single- and multi-signal data for all three SVM kernels.

In summary, fNIRS signals were successfully assessed to evaluate olfactory stimulation, and simple extracted features were employed for SVM training with three kernel functions to assess valuable data.

Author Contributions: Conceptualization, C.-H.C. and P.-L.L.; methodology, C.-H.C. and C.-K.L.; software, C.-H.C.; validation, C.-H.C., C.-K.L. and C.-W.J.; formal analysis, C.-H.C. and C.-K.L.; investigation, C.-H.C.; resources, P.-L.L. and K.-K.S.; data curation, C.-H.C. and C.-K.L.; writingoriginal draft preparation, C.-H.C. and C.-K.L.; writing-review and editing, C.-H.C., C.-K.L. and C.-W.J.; visualization, C.-H.C. and C.-K.L.; supervision, P.-L.L. and K.-K.S.; project administration, P.-L.L. and K.-K.S.; All authors have read and agreed to the published version of the manuscript.

Funding: This research received no external funding.

Institutional Review Board Statement: The study was carried out in compliance with the tenets of the Helsinki declaration. All participants provided informed consent, and the study was approved by the Ethics Committee of the Institutional Review Board (project number: TYGH101058; from 1 January to 31 December 2013) of Taoyuan General Hospital, Taiwan.

Informed Consent Statement: Informed consent was obtained from all subjects involved in the study. Written informed consent has been obtained from the patient(s) to publish this paper.

Data Availability Statement: The data are not publicly available due to the privacy concern raised by our IRB. 
Acknowledgments: The authors thank all the subjects who participated in this study.

Conflicts of Interest: The authors declare no conflict of interest.

\section{References}

1. Moein, S.T.; Khoneiveh, S.; Mirmobini, S.; Wong, A.; Zakeri, I.; Pourrezaei, K. Smell detection could be traced in fNIRS signals recorded from the forehead. In Biophotonics in Exercise Science, Sports Medicine, Health Monitoring Technologies, and Wearables; SPIE: San Francisco, CA, USA, 2020; p. 1123705.

2. Kokan, N.; Sakai, N.; Doi, K.; Fujio, H.; Hasegawa, S.; Tanimoto, H.; Nibu, K.-I. Near-infrared Spectroscopy of Orbitofrontal Cortex during Odorant Stimulation. Am. J. Rhinol. Allergy 2011, 25, 163-165. [CrossRef]

3. Min, B.-K.; Marzelli, M.J.; Yoo, S.-S. Neuroimaging-based approaches in the brain-computer interface. Trends Biotechnol. 2010, 28, 552-560. [CrossRef] [PubMed]

4. Shin, J.; Müller, K.-R.; Hwang, H.-J. Near-infrared spectroscopy (NIRS)-based eyes-closed brain-computer interface (BCI) using prefrontal cortex activation due to mental arithmetic. Sci. Rep. 2016, 6, 36203. [CrossRef] [PubMed]

5. Power, S.D.; Kushki, A.; Chau, T. Automatic single-trial discrimination of mental arithmetic, mental singing and the no-control state from prefrontal activity: Toward a three-state NIRS-BCI. BMC Res. Notes 2012, 5, 141. [CrossRef] [PubMed]

6. Hong, K.-S.; Naseer, N.; Kim, Y.-H. Classification of prefrontal and motor cortex signals for three-class fNIRS-BCI. Neurosci. Lett. 2015, 587, 87-92. [CrossRef] [PubMed]

7. Lötsch, J.; Kringel, D.; Hummel, T. Machine Learning in Human Olfactory Research. Chem. Senses 2019, 44, 11-22. [CrossRef] [PubMed]

8. Bushdid, C.; De March, C.A.; Fiorucci, S.; Matsunami, H.; Golebiowski, J. Agonists of G-Protein-Coupled Odorant Receptors Are Predicted from Chemical Features. J. Phys. Chem. Lett. 2018, 9, 2235-2240. [CrossRef]

9. Gonzalez, E.; Liljenström, H.; Ruiz, Y.; Li, G. A biologically inspired model for pattern recognition. J. Zhejiang Univ. Sci. B 2010, 11, 115-126. [CrossRef]

10. Casjens, S.; Eckert, A.; Woitalla, D.; Ellrichmann, G.; Turewicz, M.; Stephan, C. Diagnostic value of the impairment of olfaction in Parkinson's disease. PLoS ONE 2013, 8, e64735. [CrossRef]

11. Ishimaru, T.; Yata, T.; Horikawa, K.; Hatanaka, S. Near-infrared spectroscopy of the adult human olfactory cortex. Acta Oto-Laryngol. 2004, 124 (Suppl. 553), 95-98. [CrossRef]

12. Kobayashi, E.; Kusaka, T.; Karaki, M.; Kobayashi, R.; Itoh, S.; Mori, N. Functional Optical Hemodynamic Imaging of the Olfactory Cortex. Laryngoscope 2007, 117, 541-546. [CrossRef]

13. Igarashi, M.; Ikei, H.; Song, C.; Miyazaki, Y. Effects of olfactory stimulation with rose and orange oil on prefrontal cortex activity. Complement. Ther. Med. 2014, 22, 1027-1031. [CrossRef]

14. Aoyama, S.; Toshima, T.; Saito, Y.; Konishi, N.; Motoshige, K.; Ishikawa, N.; Nakamura, K.; Kobayashi, M. Maternal breast milk odour induces frontal lobe activation in neonates: A NIRS study. Early Hum. Dev. 2010, 86, 541-545. [CrossRef]

15. Bartocci, M.; Winberg, J.; Ruggiero, C.; Bergqvist, L.L.; Serra, G.; Lagercrantz, H. Activation of Olfactory Cortex in Newborn Infants After Odor Stimulation: A Functional Near-Infrared Spectroscopy Study. Pediatr. Res. 2000, 48, 18-23. [CrossRef]

16. Azuma, K.; Uchiyama, I.; Tanigawa, M.; Bamba, I.; Azuma, M.; Takano, H.; Yoshikawa, T.; Sakabe, K. Assessment of cerebral blood flow in patients with multiple chemical sensitivity using near-infrared spectroscopy—recovery after olfactory stimulation: A case-control study. Environ. Health Prev. Med. 2015, 20, 185-194. [CrossRef]

17. Azuma, K.; Uchiyama, I.; Tanigawa, M.; Bamba, I.; Azuma, M.; Takano, H.; Yoshikawa, T.; Sakabe, K. Association of Odor Thresholds and Responses in Cerebral Blood Flow of the Prefrontal Area during Olfactory Stimulation in Patients with Multiple Chemical Sensitivity. PLoS ONE 2016, 11, e0168006. [CrossRef]

18. Azuma, K.; Uchiyama, I.; Takano, H.; Tanigawa, M.; Azuma, M.; Bamba, I.; Yoshikawa, T. Changes in Cerebral Blood Flow during Olfactory Stimulation in Patients with Multiple Chemical Sensitivity: A Multi-Channel Near-Infrared Spectroscopic Study. PLoS ONE 2013, 8, e80567. [CrossRef] [PubMed]

19. Schönle, P.; Fateh, S.; Burger, T.; Huang, Q. A power-efficient multi-channel PPG ASIC with 112dB receiver DR for pulse oximetry and NIRS. In Proceedings of the 2017 IEEE Custom Integrated Circuits Conference (CICC), Austin, TX, USA, 30 April-3 May 2017; pp. 1-4.

20. Matcher, S.; Elwell, C.; Cooper, C.; Cope, M.; Delpy, D. Performance Comparison of Several Published Tissue Near-Infrared Spectroscopy Algorithms. Anal. Biochem. 1995, 227, 54-68. [CrossRef]

21. Watanabe, E.; Yamashita, Y.; Maki, A.; Ito, Y.; Koizumi, H. Non-invasive functional mapping with multi-channel near infra-red spectroscopic topography in humans. Neurosci. Lett. 1996, 205, 41-44. [CrossRef]

22. Kranjec, J.; Beguš, S.; Geršak, G.; Drnovšek, J. Non-contact heart rate and heart rate variability measurements: A review. Biomed. Signal Process. Control. 2014, 13, 102-112. [CrossRef]

23. Mengelkoch, L.J.; Martin, D.; Lawler, J. A Review of the Principles of Pulse Oximetry and Accuracy of Pulse Oximeter Estimates During Exercise. Phys. Ther. 1994, 74, 40-49. [CrossRef] [PubMed]

24. Allen, J. Photoplethysmography and its application in clinical physiological measurement. Physiol. Meas. 2007, 28, R1. [CrossRef] [PubMed]

25. Tamura, T.; Maeda, Y.; Sekine, M.; Yoshida, M. Wearable Photoplethysmographic Sensors—Past and Present. Electronics 2014, 3 , 282-302. [CrossRef] 
26. Mendelson, Y. Pulse Oximetry: Theory and Applications for Noninvasive Monitoring. Clin. Chem. 1992, 38, 1601-1607. [CrossRef]

27. Nitzan, M.; Noach, S.; Tobal, E.; Adar, Y.; Miller, Y.; Shalom, E.; Engelberg, S. Calibration-Free Pulse Oximetry Based on Two Wavelengths in the Infrared-A Preliminary Study. Sensors 2014, 14, 7420-7434. [CrossRef]

28. Buxton, R.B.; Uludağ, K.; Dubowitz, D.J.; Liu, T.T. Modeling the hemodynamic response to brain activation. NeuroImage 2004, 23, S220-S233. [CrossRef]

29. Huppert, T.; Hoge, R.; Diamond, S.; Franceschini, M.; Boas, D. A temporal comparison of BOLD, ASL, and NIRS hemodynamic responses to motor stimuli in adult humans. NeuroImage 2006, 29, 368-382. [CrossRef]

30. Strangman, G.; Culver, J.P.; Thompson, J.H.; Boas, D.A. A quantitative comparison of simultaneous BOLD fMRI and NIRS recordings during functional brain activation. Neuroimage 2002, 17, 719-731. [CrossRef]

31. Liao, L.-D.; Lin, C.-T.; Shih, Y.-Y.I.; Lai, H.-Y.; Zhao, W.-T.; Duong, T.Q.; Chang, J.-Y.; Chen, Y.-Y.; Li, M.-L. Investigation of the cerebral hemodynamic response function in single blood vessels by functional photoacoustic microscopy. J. Biomed. Opt. 2012, 17, 0612101. [CrossRef]

32. Villringer, A.; Planck, J.; Hock, C.; Schleinkofer, L.; Dirnagl, U. Near infrared spectroscopy (NIRS): A new tool to study hemodynamic changes during activation of brain function in human adults. Neurosci. Lett. 1993, 154, 101-104. [CrossRef]

33. Delpy, D.T.; Cope, M.; Van Der Zee, P.; Arridge, S.; Wray, S.; Wyatt, J. Estimation of optical pathlength through tissue from direct time of flight measurement. Phys. Med. Biol. 1988, 33, 1433-1442. [CrossRef]

34. Hoshi, Y.; Onoe, H.; Watanabe, Y.; Andersson, J.; Bergström, M.; Lilja, A.; Långstöm, B.; Tamura, M. Non-synchronous behavior of neuronal activity, oxidative metabolism and blood supply during mental tasks in man. Neurosci. Lett. 1994, 172, 129-133. [CrossRef]

35. Shiffler, R.E. Maximum Z scores and outliers. Am. Stat. 1988, 42, 79-80.

36. Jrad, N.; Congedo, M.; Phlypo, R.; Rousseau, S.; Flamary, R.; Yger, F.; Rakotomamonjy, A. sw-SVM: Sensor weighting support vector machines for EEG-based brain-computer interfaces. J. Neural Eng. 2011, 8, 056004. [CrossRef]

37. Abibullaev, B.; An, J. Classification of frontal cortex haemodynamic responses during cognitive tasks using wavelet transforms and machine learning algorithms. Med. Eng. Phys. 2012, 34, 1394-1410. [CrossRef]

38. Muller, K.-R.; Mika, S.; Ratsch, G.; Tsuda, K.; Schoelkopf, B. An introduction to kernel-based learning algorithms. IEEE Trans. Neural Netw. 2001, 12, 181-201. [CrossRef]

39. Van Stralen, K.J.; Stel, V.S.; Reitsma, J.B.; Dekker, F.W.; Zoccali, C.; Jager, K.J. Diagnostic methods I: Sensitivity, specificity, and other measures of accuracy. Kidney Int. 2009, 75, 1257-1263. [CrossRef]

40. Hanley, J.A.; McNeil, B.J. The meaning and use of the area under a receiver operating characteristic (ROC) curve. Radiology 1982, 143, 29-36. [CrossRef] [PubMed]

41. Fawcett, T. An introduction to ROC analysis. Pattern Recognit. Lett. 2006, 27, 861-874. [CrossRef]

42. Moritz, S.; Kasprzak, P.; Arlt, M.; Taeger, K.; Metz, C. Accuracy of cerebral monitoring in detecting cerebral ischemia during carotid endarterectomya comparison of transcranial doppler sonography, near-infrared spectroscopy, stump pressure, and somatosensory evoked potentials. J. Am. Soc. Anesthesiol. 2007, 107, 563-569. [CrossRef] [PubMed]

43. Karamzadeh, N.; Amyot, F.; Kenney, K.; Anderson, A.; Chowdhry, F.; Dashtestani, H.; Wassermann, E.M.; Chernomordik, V.; Boccara, C.; Wegman, E.; et al. A machine learning approach to identify functional biomarkers in human prefrontal cortex for individuals with traumatic brain injury using functional near-infrared spectroscopy. Brain Behav. 2016, 6, e00541. [CrossRef] [PubMed]

44. Calamanti, C.; Moccia, S.; Migliorelli, L.; Paolanti, M.; Frontoni, E. Learning-Based Screening of Endothelial Dysfunction From Photoplethysmographic Signals. Electronics 2019, 8, 271. [CrossRef]

45. Price, J. Olfactory Higher Centers Anatomy. In Encyclopedia of Neuroscience; Elsevier Ltd.: Amsterdam, The Netherlands, 2009; pp. 129-136.

46. Rolls, E.T. The functions of the orbitofrontal cortex. Brain Cogn. 2004, 55, 11-29. [CrossRef]

47. Wenning, G.K.; Shephard, B.; Hawkes, C.; Petruckevitch, A.; Lees, A.; Quinn, N. Olfactory function in atypical parkinsonian syndromes. Acta Neurol. Scand. 1995, 91, 247-250. [CrossRef]

48. Zou, Y.M.; Da Lu, L.P.L.; Zhang, H.H.; Zhou, Y.Y. Olfactory dysfunction in Alzheimer's disease. Neuropsychiatr. Dis. Treat. 2016, 12, 869. [CrossRef]

49. Murphy, C.; Schubert, C.R.; Cruickshanks, K.J.; Klein, B.E.K.; Klein, R.; Nondahl, D.M. Prevalence of Olfactory Impairment in Older Adults. JAMA 2002, 288, 2307-2312. [CrossRef]

50. Hummel, T.; Nordin, S. Olfactory disorders and their consequences for quality of life. Acta Oto-Laryngol. 2005, 125, 116-121. [CrossRef]

51. Doty, R.L.; Kamath, V. The influences of age on olfaction: A review. Front. Psychol. 2014, 5, 20. [CrossRef] 